\title{
Actitudes lingüísticas hacia el quechua y sus hablantes en la Universidad Nacional Mayor de San Marcos
}

\section{Linguistic attitudes towards Quechua and its speakers at the National University of San Marcos}

\author{
Angela Sullón Rios \\ Universidad Nacional Mayor de San Marcos \\ angela.sullon@unmsm.edu.pe
}

\begin{abstract}
Resumen
El presente estudio exploratorio revela las actitudes lingüísticas que reflejan los estudiantes de la Universidad Nacional Mayor de San Marcos hacia la presencia del quechua en dicha universidad. El objetivo de esta investigación es analizar la valoración que los estudiantes de la UNMSM tienen hacia la lengua y sus hablantes. Se trabajó con una muestra no probabilística de 20 estudiantes, uno de cada facultad, a quienes se aplicó un cuestionario que mide los enunciados a través de la escala de Likert. La investigación concluye que los estudiantes muestran actitudes lingüísticas positivas ante el quechua en el contexto académico.
\end{abstract}

Palabras clave: actitud lingüística, quechua, identidad, discriminación lingüística, UnMSM.

\begin{abstract}
The present study reveals the linguistic attitudes shown by the students of the National University of San Marcos (UNMSM) towards Quechua at same university. The objective of this research is to analyse the assessment that UNMSM students have towards the language and its speakers. I work with a non-probabilistic sample of 20 students, one from each faculty. A questionnaire that measures the statements through the Likert scale was applied on them. The research concludes that students show positive linguistic attitudes towards Quechua in the academic context.
\end{abstract}

Keywords: linguistic attitude, Quechua, identity, linguistic discrimination, UnMSM.

Recibido: 20.06.19

Aceptado: 15.08 .19 


\section{Introducción}

En el Perú, por décadas, la migración se dio sobre todo por parte de la población andina, quienes dejaban sus tierras y costumbres, en busca de una mejor educación, un mejor trabajo, una mejor vida. Muchos de ellos, monolingües de quechua, sufrieron discriminación racial y lingüística por parte de los limeños, e incluso aquellos que eran bilingües, a causa del motoseo. Es en el plano de la educación donde por décadas se ha visto la discriminación lingüística hacia los estudiantes que provienen de la zona andina, e incluso la negación de la verdadera identidad, por el simple hecho de que sus familias migraron y ellos nacieron en Lima. Por esta razón, es el contexto educativo el más ideal para revelar las actitudes lingüísticas que los estudiantes puedan presentar hacia los estudiantes quechuahablantes, así como hacia el quechua como parte de su contexto educativo.

En los últimos años, el Perú ha cobrado interés en las actitudes lingüísticas (Dueñas) de las distintas comunidades del país, revelando actitudes positivas o negativas que influyen de manera importante en la lealtad hacia la lengua o en su desplazamiento y posterior muerte. Es con el conocimiento de estas actitudes que se pueden realizar proyectos de revitalización lingüística, de fortalecimiento de la identidad, como parte de las políticas lingüísticas del Estado. Asimismo, existen investigaciones que se abordan desde el contexto educativo, como la de Kenfield, Huayllani y Huillca en el 2017 titulado Actitudes lingüísticas de estudiantes universitarios hacia el quechua de Cusco, donde se estudiaron las actitudes lingüísticas de estudiantes de la Universidad Nacional San Antonio Abad del Cusco (UnSAAC) hacia el quechua, siendo esta una de las universidades con más población estudiantil de quechuahablantes. Esta investigación se realizó a través de una encuesta anónima no probabilística a más de 300 alumnos durante el 2016. Se comprobó la hipótesis que apuntaba a que dichos estudiantes presentan actitudes lingüísticas positivas hacia el quechua tanto dentro como fuera de la universidad.

Otro de estos estudios, es la tesis de Maestría presentada por Vizcarra en el 2012 titulada Actitudes lingüísticas hacia las lenguas andinas por los estudiantes de la Facultad de Ciencias Sociales de la U. N. A. Puno, cuyo objetivo era determinar las actitudes lingüísticas que los estudiantes de dicha facultad presentan ante las lenguas andinas. El diseño de investigación es cualitativo y fue observado desde la hermenéutica para la interpretación de los datos. La investigación obtuvo como resultado, que tanto los estudiantes bilingües como los monolingües del español presentan actitud cognitiva y conductual positiva hacia las lenguas andinas, ya que representa su identidad cultural. Estas lenguas son empleadas tanto dentro

100 Lengua \& Sociedad, vol. 18, n. ${ }^{\circ}$ 1, Lima, enero-junio de 2019 
de las comunidades como en los mismos hogares, sin embargo, encuentra restricción en la ciudad y dentro de la universidad, por ser el castellano la lengua hegemónica del Perú.

Por consiguiente, el presente estudio exploratorio tiene por objetivo revelar a través de la escala de Likert la perspectiva de valoración que muestran los estudiantes ante cada enunciado, de esta manera se evidenciará las actitudes lingüísticas que presentan los estudiantes de la Universidad Nacional Mayor de San Marcos hacia el quechua y sus hablantes.

El presente estudio se estructura de la siguiente manera. En el apartado uno se presenta la introducción, donde se expone los antecedentes, el objetivo y la estructura de la investigación. En el apartado dos se desarrolla el marco teórico con conceptos como cultura, identidad cultural, bilingüismo, migración, actitudes lingüísticas y el enfoque mentalista. En el apartado tres se presenta la metodología empleada para el desarrollo del trabajo. En el apartado cuatro, el análisis de la información obtenida a través de los cuestionarios. Finalmente, en el apartado cinco se presentan las conclusiones.

\section{Bilingüismo}

Se conceptualizará al bilingüismo según la propuesta de Baker, quien afirma que para poder definir al bilingüismo se debe tener en cuenta las dimensiones y distinciones más importantes para definir quién es o no bilingüe. En este sentido, la distinción inicial parte del bilingüismo como fenómeno individual y bilingüismo como posesión social o de grupo. En cuanto al nivel individual la distinción esencial es entre capacidad y uso.

Ahora bien, Baker propone cuatro capacidades lingüísticas básicas: escuchar (comprensión auditiva), hablar, leer y escribir. Sin embargo, el autor sugiere que dichas capacidades pueden desarrollarse en mayor y menor medida; es decir, las cuatro capacidades básicas pueden recomponerse en subescalas y dimensiones, «hay destrezas dentro de las destrezas» (p.32).

En cuanto al uso individual de un bilingüe, Baker enfatiza en la importancia de considerar el dominio y el contexto. A medida que un bilingüe se mueve de una situación a otra, así puede cambiar la lengua en términos de tipo (por ejemplo, español, francés, irlandés), contenido (por ejemplo, vocabulario) y estilo. A lo largo del tiempo y del lugar, las dos lenguas de un individuo nunca son estáticas sino que cambian y evolucionan. 


\section{El quechua y la UNMSM}

El Banco de Datos de Pueblos Indígenas u Originarios, señala que el quechua es la lengua originaria más empleada en el Perú. Según el censo realizado en el 2017 a nivel nacional, la población que se autopercibe de origen quechua se trataría de 5’176,809 personas. El quechua no solo es hablado en Perú, sino también en otros 6 países de América del Sur, como son Ecuador, Colombia, Bolivia, Argentina, Chile y Brasil. En el Perú, y seguramente en los otros países también, se presentan variedades de quechua, esto es por su historia y geografía. Es por ello que en la actualidad, podemos distinguir hasta nueve variedades distribuidos en el norte, centro y sur del país, donde ha tenido contacto con otras lenguas. Debemos saber que los hablantes de esta lengua son denominados quechua hablantes; sin embargo, ser quechua hablante, significa mucho más que eso, ya que es identidad, costumbres, tradiciones, historia, etcétera.

Por años, miles de migrantes de las zonas andinas, muchos de ellos quechua hablantes, o bilingües con ragos de motoseo, se asentaron en Lima en busca de una mejor vida. Es así como en el contexto universitario, el informe final de la Comisión de la Verdad y Reconciliación Nacional publicado en el 2003, nos revela que en el año 1970 la Universidad Nacional Mayor de San Marcos albergó a 19,461 estudiantes, en su mayoría inmigrantes provenientes de la zona andina, los que buscaban integrarse a dicha universidad como un medio de movilidad social. Aunque no hayan estudios cuantitativos, este dato se confirma por el hecho de que, al exterior de Lima solo existían 17 universidades, muchas de ellas con menos de 5 años de funcionamiento.

\section{Actitudes lingüísticas y el enfoque mentalista}

Existen diferentes conceptos cuando se habla acerca de actitudes lingüísticas, entre ellas tenemos la de Baker (1992), quien afirma que las actitudes lingüísticas son predisposiciones asimiladas; en otras palabras, no se heredan ni se transfieren a través de los genes. Así también, López Morales afirma que solo existen actitudes positivas o negativas, mas no una neutral, y en los casos donde no se reconozca alguna de estas, significa que hay ausencia de actitud. Además, López trabaja la actitud lingüística como una definición componencial, es decir, la suma del valor cognitivo, afectivo y conativo (Citado en Dueñas, 2016, p.21).

Uno de los enfoques teóricos que se trabajan al tratar las actitudes lingüísticas es el mentalista. Fishman (1970) sostiene que la actitud es tomada como un estado

102 Lengua \& Sociedad, vol. 18, n. ${ }^{\circ}$ 1, Lima, enero-junio de 2019 
mental interior, siendo esta la variable que aparece ante un estímulo recibido por el sujeto y concluye con una respuesta (Citado en González, 2008, p.230).

González (2008) manifiesta que aunque este enfoque no nos permita realizar una observación directa de las actitudes de los sujetos con los que se trabajarán, puesto que se asume como un estado mental, sí nos permitirá predecir, es decir, podremos trabajar con patrones sistemáticos. Finalmente, Erdösová (2011) expresa que la intención del enfoque mentalista es revelar la forma de pensar del hablante, mas no la de su actuar (Citado en Dueñas, 2016, p. 24).

\section{Metodología}

La metodología que se empleó en este estudio es de tipo cuantitativa, ya que se medirá la frecuencia en las valoraciones que presenten los estudiantes ante determinado enunciado. Se empleó el muestreo intencionado, pues se eligió al azar un estudiante por cada facultad, sin importar la escuela a la que pertenece, hasta completar veinte personas, pertenecientes a las veinte facultades de la Universidad Nacional Mayor de San Marcos; tantos las de Ciudad Universitaria como las externas.

Se elaboró un cuestionario de tres enunciados que miden las actitudes a través de la escala de Likert. Esta escala presenta cuatro valoraciones: a) Estoy completamente de acuerdo, b) Estoy de acuerdo, c) Estoy en desacuerdo y, d) Estoy completamente en desacuerdo. El cuestionario fue aplicado los días 18 y 19 de noviembre, del presente año. Así mismo, contiene enunciados que hacen referencia al quechua como ajeno a uno mismo, para conocer la perspectiva que tienen hacia las personas que lo emplean; así como también, enunciados que proponen las situaciones de hacer del quechua parte de uno mismo, ya sea como curso obligatorio en la carrera o en una sustentación de Tesis. Se debe mencionar que los cuatro primeros enunciados de este cuestionario fueron tomados de la investigación de Kenfield, Hayllani y Huillca (2018), titulada Actitudes lingüisticas de estudiantes universitarios hacia el quechua en Cusco. Los enunciados son los siguientes:

a. El idioma quechua está a la misma altura del castellano.

b. En general los jóvenes que saben idiomas originarios Andinos quechuas deberían usar sus idiomas entre ellos nomás y fuera de la Universidad.

c. En general los jóvenes que saben el idioma quechua deberían usarlo dentro de la Universidad para que los demás aprendan (si no lo conocen) o practiquen (si en caso lo conocen) el quechua. 
d. La universidad no debería fomentar el uso del quechua por ser un retraso.

e. Deberían integrar el quechua como curso obligatorio en las mallas curriculares de todas las carreras profesionales de la UNMSM.

f. Asistiría a clases de cualquier variedad de quechua si fueran gratuitas.

g. Se deberían replicar acciones como la reciente sustentación de Tesis escrita en quechua, en dicha lengua.

h. Los estudiantes quechuahablantes de la UNMSM son discriminados por presentar motoseo.

i. Los estudiantes de quechuahablantes de la UNMSM se sienten orgullosos de su identidad andina.

j. Un profesional monolingüe de habla castellana obtendría un mejor puesto de trabajo que un profesional bilingüe (quechua-castellano).

\section{Análisis}

\section{a. El idioma quechua está a la misma altura del castellano.}

En el primer enunciado tenemos, indudablemente un panorama positivo, pues el $40 \%$ se muestra totalmente de acuerdo con la idea de que el quechua se encuentra a la misma altura que el español; de igual modo, el $45 \%$ estuvo de acuerdo con el enunciado, lo que significaría en la actualidad, un cambio en la perpectiva de valoración hacia el quechua, considerando que por décadas, toda expresión de identidad andina era minorizada, sobre todo la lengua. En contraste a esto, el $10 \%$ se mostró en desacuerdo y solo el $5 \%$, que representa a una persona estuvo totalmente en desacuerdo.

\section{b. En general los jóvenes que saben idiomas originarios Andinos quechuas deberían usar sus idiomas entre ellos nomás y fuera de la Universidad.}

En este enunciado se revela también un panorama positivo hacia el quechua, ya que el $45 \%$ de los estudiantes que apoyaron en esta investigación se mostraron en desacuerdo con que los quechua hablantes deban hablar únicamente en espacios externos a la universidad. Así también el $35 \%$ estuvo totalmente en desacuerdo.

Por otro lado, el $10 \%$ se mostró totalmente de acuerdo; de igual forma, el $10 \%$ se mostró de acuerdo, revelando en una pequeña población, la valoración negativa y de indiferencia hacia los quechua hablantes.

104 Lengua \& Sociedad, vol. 18, n. ${ }^{\circ}$ 1, Lima, enero-junio de 2019 
c. En general los jóvenes que saben el idioma quechua deberían usarlo dentro de la Universidad para que los demás aprendan (si no lo conocen) o practiquen (si en caso lo conocen) el quechua.

Diferente a los resultados que obtuvimos en el enunciado anterior y contraditorio; en este, absolutamente todos los estudiantes mostraron actitudes positivas hacia los quechua hablantes y su lengua, ya que están a favor de que ellos puedan usar su lengua dentro de la universidad y enseñarla a los que desconocen dicha lengua. Los resultados dieron lo siguiente: el $60 \%$ de la población se mostró totalmente de acuerdo con el enunciado y el $40 \%$ estuvo de acuerdo.

d. La universidad no debería fomentar el uso del quechua por ser un retraso.

Este enunciado nos presenta un panorama positivo, donde los estudiantes consideran que la universidad debería fomentar el quechua, ya que en su opinión no se trata de un retraso para ellos. En los resultados, el $75 \%$ de la población se mostró totalmente en desacuerdo con este enunciado, también el $20 \%$ se mostró de acuerdo, y solo el $5 \%$ estuvo de acuerdo con no fomentar el quechua por ser un retraso..

e. Deberían integrar el quechua como curso obligatorio en las mallas curriculares de todas las carreras profesionales de la UNMSM.

En el primer enunciado se revela que el $50 \%$ de los estudiantes de la Universidad Nacional Mayor de San Marcos estaría de acuerdo con añadir a sus mallas curriculares, el quechua como curso obligatorio. Esto, ocurre probablemente porque al egresar algunas carreras como Sociología, Ingeniería de Minas, entre otras, solicita que sus trabajadores dominen esta lengua, sobre todo cuando el trabajo se realizará en provincia. El $15 \%$ de los veinte estudiantes estuvo totalmente de acuerdo. En contraste, el $20 \%$ de los estudiantes se mostraron en desacuerdo y el $15 \%$ estuvo absolutamente en desacuerdo.

f. Asistiría a clases de cualquier variedad de quechua si fueran gratuitas. En este enunciado el $50 \%$ de los estudiantes se muestran totalmente de acuerdo hacia el aprendizaje gratuito de cualquier variedad de quechua. Así también el $40 \%$ de los estudiantes se mostraron de acuerdo a este enunciado. Probablemente, porque en la actualidad, el quechua ha sido reconocido por las universidades como una lengua con la que se puede sustentar una tesis, prestándole igual peso que a las lenguas extranjeras. Esto es absolutamente positivo para el quechua en los espacios académicos, e incluso para hacer del quechua una lengua académica. 
g. Se deberían replicar acciones como la reciente sustentación de Tesis escrita en quechua, en dicha lengua.

Este enunciado presenta cifras muy parecidas al enunciado anterior, donde el $50 \%$ de los estudiantes se muestra totalmente de acuerdo y el $40 \%$ de ellos se muestra de acuerdo con replicar acciones como la reciente sustentación de Tesis escrita en quechua. Sin duda, acciones como esta nos muestran el potencial valor académico que puede poseer el quechua, pero que aún no se trabaja como debería.

En contraste el $15 \%$ se muestra en desacuerdo y el $15 \%$ se muestra totalmente en desacuerdo, siendo una cifra muy poco considerable.

\section{h. Los estudiantes quechua hablantes de la UNMSM son discriminados por presentar motoseo.}

Los resultados de este enunciado nos presenta un panorama muy interesante, ya que el $50 \%$ de los estudiantes se mostró de acuerdo con la premisa, lo que indica que aún se siguen presentando casos de discriminación lingüística. En contraste a esto, tenemos que el 40 \% de la población en estudio se mostró en desacuerdo, indicando que los estudiantes quechua hablantes no son discriminados por presentar motoseo, lo que nos revela una actitud positiva de parte de estos estudiantes, desde la perspectiva de ellos mismos y hacia los demás. Por otro lado las premisas extremas, es decir, totalmente en de acuerdo y totalmente en desacuerdo obtuvieron solo un voto cada uno, lo que representa el $5 \%$ del total, en cada nivel de valoración.

\section{i. Los estudiantes de quechua hablantes de la UNMSM se sienten orgullosos de su identidad andina.}

Este enunciado tiene resultados realmente curiosos, ya que en la premisa anterior, tuvimos la mitad a favor y casi la otra mitad en contra; sin embargo aquí, los votos en su gran mayoría se muestran de acuerdo (65\% de ellos se mostró de acuerdo y el $15 \%$ se mostró totalmente de acuerdo) en que los estudiantes que provienen de las zonas andinas se sienten orgullosos de su identidad andina. Revelando el pensamiento de los estudiantes, que, a pesar de que los estudiantes quechua hablantes puedan ser discriminados por presentar motoseo, ellos se siguen sintiendo orgullosos de su identidad andina. Nadie estuvo totalmente en desacuerdo y solo el $20 \%$ se mostró en desacuerdo.

106 Lengua \& Sociedad, vol. 18, n. ${ }^{\circ}$ 1, Lima, enero-junio de 2019 
j. Un profesional monolingüe de habla castellana obtendría un mejor puesto de trabajo que un profesional bilingüe (quechua-castellano).

Los resultados de este enunciado nos revela una valoración postiva hacia el quechua, descartando que un monolingüe de habla castellana, pueda obtener un mejor puesto de trabajo que un hablante bilingüe de quechua castellano, donde incluso este, probablemente puede presentar motosidad. Los resultados presentan los siguientes porcentajes: el $65 \%$ de los estudiantes que apoyaron en la investigación se mostraron en desacuerdo, así mismo, el $20 \%$ estuvo totalmente en desacuerdo con dicho enunciado. En contraste, el $10 \%$ se mostró totalmente de acuerdo y el $5 \%$ de acuerdo, en que por ser profesionales bilingües no podrían obtener un mejor puesto de trabajo que un profesional monolingüe del español.

\section{Conclusiones}

El análisis de los datos nos revela que los estudiantes de la Universidad Nacional Mayor de San Marcos presentan actitudes lingüísticas positivas hacia los quechua hablantes y su lengua, lo que significa que están quedando atrás esos años de discriminación lingüística, donde estamos dispuestos a conocer al otro y a nosotros mismos. El quechua tal y como se ha presentado en las premisas, hace referencia al ámbito académico, donde en su mayoría, los estudiantes estuvieron de acuerdo con incluirlo en sus mallas académicas de manera obligatoria, así como el de llevarlo como un curso extracurricular de manera gratuita; esto también nos demuestra la valoración positiva que se presenta con la lengua desde la perspectiva de su utilidad y uso académico. En cuanto a los estudiantes quechua hablantes, la población en estudio reveló que a pesar de que sigan siendo testigos de acciones discriminadoras hacia los estudiantes que presentan motoseo, ellos piensan que los estudiantes quechua hablantes sí se sienten orgullosos de su identidad andina. 


\section{Referencias bibliográficas}

Baker, C. (2000). Fundamentos de Educación Bilingüe y Bilingüismo. España: Cátedra.

Comisión de la Verdad y Reconciliación. (2003). Informe Final (Tomo V). Lima: Perú. Recuperado de http://idehpucp.pucp.edu.pe/images/docs/tomo_v.pdf

Dueñas, E. (2016). Actitudes en situación de contacto lingüístico en la comunidad nativa «Siete de junio», Oxapampa (Tesis de licenciatura). Universidad Nacional Mayor de San Marcos, Lima.

González, J. (2008). Metodología para el estudio de las actitudes lingüísticas. Actas del XXXVII Simposio Internacional de la Sociedad Española de Lingüística (SEL). Recuperado de http://www.unav.es/linguis/simposiosel/actas/

Guzmán, E. (2005). Logros y retos del Frente Indígena Oaxaqueño Binacional: una organización para el futuro de los migrantes indígenas (Tesis de licenciatura). Universidad de las Américas Puebla, México.

Kenfield, Y., Huayllani, W., \& Huillca, E. (2018). Actitudes lingüísticas de estudiantes universitarios hacia el quechua en Cusco. Revista de investigación Apuntes Universitarios, 8(1). Recuperado de https://revistas.upeu.edu.pe/index.php/ ra_universitarios/article/view/122

Ministerio de Cultura. (s. f.). Quechua. Base de Datos de Pueblos Indígenas u Originarios. Recuperado de https://bdpi.cultura.gob.pe/lenguas/quechua

Ramírez, N. (2015). Percepciones sobre interculturalidad (Tesis Doctoral). Universidad de Valladolid, Valladolid.

Rondán, O. (2014). Actitudes lingüísticas de los estudiantes de la Escuela Académico Profesional de Educación de la UnASAm ante la lengua quechua y el español - 2013. Recuperado de http://repositorio.unasam.edu.pe/bitstream/ handle/unasam/2298/to33_31619094_ti.pdf?sequence $=1$ \&isallowed $=y$

Vicerrectorado Académico de Pregrado. (s. f.). Áreas académico profesionales. Vicerrectorado Académico de Pregrado. Recuperado de https://viceacademico. unmsm.edu.pe/?page_id $=5580$

Vizcarra, F. (2012). Actitudes Lingüísticas hacia las lenguas andinas por los estudiantes de la Facultad de Ciencias Sociales de la UNA Puno (Tesis de maestría). Universidad Nacional del Altiplano, Puno. 